\title{
Resistencia al cizallamiento de la línea de cola en madera de tornillo (Cedrelinga cateniformis Ducke) procedente de plantaciones en la zona de Ucayali, Perú
}

\author{
Shear strength of the glue line in tornillo wood (Cedrelinga cateniformis Ducke) \\ from plantations in the Ucayali region, Peru
}

\author{
Alvaro Guerra Miranda ${ }^{1}$ y Neptalí R. Bustamante Guillén ${ }^{2}$
}

\begin{abstract}
Resumen
En este estudio se determinó la resistencia al cizallamiento de la línea de cola en uniones encoladas con polivinilo (PVA) en madera tornillo (Cedrelinga cateniformis Ducke), utilizando un encolado doble, dos presiones de prensado $\left(5\right.$ y $\left.10 \mathrm{~kg} / \mathrm{cm}^{2}\right)$ y tres extendidos $\left(150,200\right.$ y $\left.250 \mathrm{~g} / \mathrm{m}^{2}\right)$. Los resultados permitieron determinar que la resistencia promedio al cizallamiento en la línea de cola alcanza valores ligeramente inferiores a los de la madera solida (88,40 por ciento tornillo de fajas y 97,50 por ciento para tornillo de campo abierto). De la misma manera, el incremento de la presión de prensado y el extendido de cola tienen un efecto favorable en la resistencia al cizallamiento en madera tornillo de campo abierto. Asimismo, la variación de la presión de prensado y el extendido de cola no tienen influencia sobre la resistencia al cizallamiento de madera tornillo en fajas de $5 \mathrm{~m}$. Finalmente, las condiciones más favorables que permiten obtener uniones con una mayor resistencia y mayor porcentaje de madera fallada son: una presión de encolado de $10 \mathrm{~kg} / \mathrm{cm}^{2}$ para tornillo de fajas y campo abierto; y un extendido de 150 y $200 \mathrm{~g} / \mathrm{m}^{2}$ para fajas y campo abierto, respectivamente.
\end{abstract}

Palabras clave: resistencia al cizallamiento; madera fallada; extendido del adhesivo; presión.

\begin{abstract}
The shear strength of glue line at joints glued with polyvinyl (PVA) in "tornillo" wood (Cedrelinga cateniformis Duck) was determined in this research using double gluing, two press pressures $\left(5\right.$ and $\left.10 \mathrm{~kg} / \mathrm{cm}^{2}\right)$ and three spreading $\left(150,200\right.$ and $\left.250 \mathrm{~g} / \mathrm{m}^{2}\right)$. The results indicate that the average shear strength for the glue line is slightly lower than for solid wood (88,40 percent for "tornillo" plantations in strips and 97,50 percent for "tornillo" open field plantations). Likewise, increasing the press pressure and the spreading degree is beneficial for the shear strength in "tornillo" open field plantations. Similarly, a variation in the press pressure and spreading has no influence on the shear strength of "tornillo" wood coming for plantations in $5 \mathrm{~m}$ strips. Finally, the best conditions to obtain joints with greater shear strength and greater wood failure percentage are: gluing pressure of $10 \mathrm{~kg} / \mathrm{cm}^{2}$ for "tornillo" plantations in strips and in open field; and a spreading of 150 and $200 \mathrm{~g} / \mathrm{m}^{2}$ for tornillo" plantations in strips and in open field, respectively.
\end{abstract}

Key words: shear strength; wood failure; extended adhesive; pressure.

${ }^{1}$ Consultor independiente, Perú.

${ }^{2}$ Facultad de Ciencias Forestales, Universidad Nacional Agraria La Molina, Perú. E-mail: nbustamante@lamolina.edu.pe 


\section{Introducción}

En el Perú existen plantaciones forestales experimentales que se constituyen en fuente de conocimiento para el establecimiento de cultivos que permitan abastecer de materia prima la industria forestal. Las plantaciones forestales de tornillo en el Centro Experimental Alexander von Humboldt reportan buenos resultados de crecimiento y productividad (Flores et al. 2004). Villa (2009) y Vega (2011) recomiendan el uso de la madera de esta especie en mueblería y carpintería no estructural, tanto para tornillo en fajas como para campo abierto.

Aróstegui (1982) afirma que la madera tornillo es de excelente calidad; el duramen recién cortado es rosado claro y la albura blanco amarillenta; cuando seca, el duramen es de color claro castaño pálido, marcado con líneas de color oscuro que destacan sobre el fondo más claro; de grano recto a entrecruzado y de textura gruesa. El duramen es poco susceptible al ataque de agentes biológicos.

Flores et al. (2004) indica que, en plantaciones experimentales en el Bosque Nacional Alexander von Humboldt, los resultados obtenidos a 20 años de edad muestran el gran potencial de tornillo, como la especie con el mejor crecimiento y productividad tanto en fajas de enriquecimiento como en campo abierto. En plantaciones a campo abierto se determinó un DAP (diámetro promedio a la altura del pecho) de $35,80 \mathrm{~cm}$ (IMA $1,96 \mathrm{~cm} /$ año), una altura total promedio de $26,40 \mathrm{~m}$ (IMA $1,42 \mathrm{~m} / \mathrm{año}$ ), un área basal de $30,35 \mathrm{~m}^{2} /$ ha y un volumen promedio de $380,20 \mathrm{~m}^{3} / \mathrm{ha}$ (IMA $21,40 \mathrm{~m}^{3} / \mathrm{ha} /$ año), resultados que son muy superiores a los de otras especies nativas.

La madera tornillo procedente de las plantaciones del Bosque Nacional von Humboldt, según Villa (2009) y Vega (2011), es de baja densidad y resistencia mecánica. Ambos autores coinciden en asignarle los siguientes usos: mueblería, carpintería de obra no estructural, cajonería liviana, molduras y revestimiento.

Según López (1976), las propiedades de las uniones encoladas dependen principalmente de las propiedades físico-mecánicas, geometría de la unión y de las condiciones ambientales.
En las pruebas de uniones encoladas, además de la madera y el adhesivo, también debe evaluar la técnica de encolado, principalmente la extensión, presión de prensado, temperatura y preparación de la superficie.

Para la madera de quinilla colorada $(\mathrm{Hu}$ miriastrum excelsum), Takahashi (1967) encontró una resistencia al cizallamiento de $155,40 \mathrm{~kg} / \mathrm{cm}^{2}$, alcanzando valores cercanos a los de la madera sólida con el uso de cola caseína, encolado doble (de ambas superficies), extendido de $400 \mathrm{~g} / \mathrm{m}^{2}$ y presión de prensado $10 \mathrm{~kg} / \mathrm{cm}^{2}$. De la misma manera, afirma que la resistencia con un encolado doble es mayor que con el encolado simple a un mismo gramaje. El mismo autor agrega que una alta dureza de lados dificulta la aplicación uniforme de la presión de prensado, recomendando el encolado de superficies lisas y libres de ondulaciones o irregularidades.

En ensayos con madera "asta de venado" (Tabebuia capitata), Acevedo (1973) registró una resistencia al cizallamiento de $160 \mathrm{~kg} /$ $\mathrm{cm}^{2}$ empleando resorcinol-formaldehido con presión de prensado de $15 \mathrm{~kg} / \mathrm{cm}^{2}$ y un extendido de $250 \mathrm{~g} / \mathrm{m}^{2}$, siendo similares los valores de resistencia en el encolado doble y simple.

Según Brandán (1991), empleando la prensa tipo "C", se obtienen los mejores resultados en maderas de Bombacáceas (Ceiba samauma, Erioteca globosa, Matisia bicolor y Matisia cordata) con una resistencia promedio al cizallamiento de $44,70 \mathrm{~kg} / \mathrm{cm}^{2}$ y madera fallada de 62,70 por ciento. También reporta valores similares de resistencia para extendidos de cola de 150,200 y $250 \mathrm{~g} / \mathrm{m}^{2}$.

Gonzales (2006), utilizando un extendido de cola de $150 \mathrm{~g} / \mathrm{m}^{2}$ y una presión de prensado de $10 \mathrm{~kg} / \mathrm{cm}^{2}$, reportó para el pino chuncho (Schizolobium amazonicum) una resistencia al cizallamiento de $105,60 \mathrm{~kg} / \mathrm{cm}^{2}$ con 22 por ciento de madera fallada. En el caso de yanavara (Pollalesta discolor) registró una resistencia al cizallamiento de $133,10 \mathrm{~kg} / \mathrm{cm}^{2}$ con 28 por ciento de madera fallada. $\mathrm{Al}$ respecto, la misma autora afirma que existe una relación inversa entre la rugosidad superficial y la resistencia al cizallamiento. 
Es necesario complementar los estudios realizados en la madera tornillo procedente de plantaciones experimentales del bosque Alexander von Humboldt, determinando la resistencia de uniones encoladas, con la finalidad de potenciar una mejor utilización de la madera tornillo para la industria del mueble.

En la presente investigación se determinó la resistencia al cizallamiento de la línea de cola con PVA (acetato de polivinilo) en madera de tornillo procedente de plantaciones de 20 y 22 años bajo dos tratamientos silviculturales en la zona de Ucayali, Perú. De la misma manera, se determinaron las mejores condiciones de encolado que satisfagan requisitos de resistencia y calidad según tratamiento silvicultural.

\section{Materiales y Métodos}

\section{Lugar de ejecución}

La presente investigación se realizó en los Laboratorios de Propiedades Físicas y Mecánicas de la Madera, y de Pulpa y Papel del Departamento de Industrias Forestales de la Facultad de Ciencias Forestales de la Universidad $\mathrm{Na}$ cional Agraria La Molina (Lima, Perú).

\section{Procedencia del material experimental}

El material experimental procede de las plantaciones del Bosque Nacional "Alexander von Humboldt", ubicadas en el kilómetro 86 de la Carretera Federico Basadre, perteneciente a la región Ucayali (Perú). En la selección y colección de las muestras de madera se empleó la norma ASTM D5536-94 (2004).

Se seleccionaron listones de cinco (5) árboles diferentes bajo los tratamientos silviculturales de Fajas de enriquecimiento (22 años) y Campo abierto (20 años).

\section{Adhesivo}

El adhesivo utilizado es el acetato de polivinilo (PVA).

\section{Identificación de la especie}

La identificación de la especie se realizó en el Laboratorio de Anatomía de la Madera del Departamento de Industrias Forestales de la
Facultad de Ciencias Forestales de la Universidad Nacional Agraria La Molina (UNALM).

\section{Determinación del contenido de humedad de la madera}

Se determinó el contenido de humedad de la madera a través del método de estufa hasta alcanzar peso constante, según Norma Técnica Peruana NTP 251.010 (2004).

\section{Distribución de las probetas de madera}

Las probetas para el ensayo se obtuvieron a partir de los listones de 5 x 5 x $120 \mathrm{~cm}$, de acuerdo a lo especificado en el Cuadro 1.

\section{Evaluación de la superficie de la madera}

Las evaluaciones de rugosidad superficial se realizaron en probetas de $2,5 \times 5,0 \times 40,0 \mathrm{~cm}$ usando el método empleado por Meléndez y Bustamante (2003). Se empleó un rugosímetro digital que posee una aguja de sondeo cuyo movimiento sobre la superficie de la madera permite conseguir una representación numérica del perfil de la superficie analizada. Para la medición de la calidad de la superficie, se realizaron siete (7) mediciones por probeta distribuidas cada $5 \mathrm{~cm}$ (Figura 1). El parámetro de rugosidad superficial a medir fue la rugosidad media ( $\mathrm{Ra})$.

\section{Determinación del porcentaje de extractivos de la madera}

Se empleó la norma TAPPI T 204 cm-97 (1997), usando etanol como solvente de extracción. Se utilizaron muestras de 4 y 2 g de aserrín de madera tornillo para la extracción de extractivos y para la determinación el contenido de humedad, respectivamente.

\section{Evaluación del adhesivo}

Contenido de sólidos

Se utilizó el procedimiento indicado en la norma ASTM D 1489-97 (2004), utilizando tres (3) muestras de $10 \mathrm{~g}$ de adhesivo.

\section{Viscosidad}

En la determinación de la viscosidad del adhesivo se utilizó el viscosímetro Brookfield tipo aguja con spin $\mathrm{N}^{\circ} 4$, velocidad de $4 \mathrm{r} / \mathrm{min}$ a una 


\begin{tabular}{|c|c|c|}
\hline $\begin{array}{c}\text { Tratamiento } \\
\text { silvicultural }\end{array}$ & Código del árbol & $\begin{array}{c}\text { Número de } \\
\text { probetas }\end{array}$ \\
\hline \multirow{4}{*}{ Campo abierto } & 10 - F3 & 12 \\
\cline { 2 - 3 } & $9-\mathrm{F} 6$ & 12 \\
\cline { 2 - 3 } & $12-\mathrm{F} 4$ & 12 \\
\cline { 2 - 3 } & $8-\mathrm{F} 3$ & 12 \\
\hline \multirow{5}{*}{ Fajas de 5 m } & $9-\mathrm{F} 9$ & 12 \\
\cline { 2 - 3 } & $268-1$ & 12 \\
\cline { 2 - 3 } & $268-2$ & 12 \\
\cline { 2 - 3 } & 930 & 12 \\
\cline { 2 - 3 } & 274 & 12 \\
\hline & 289 & 120 \\
\hline
\end{tabular}

Cuadro 1. Distribución del número de probetas según tratamiento silvicultural.

temperatura de $23^{\circ} \mathrm{C}$, siguiendo las especificaciones técnicas del fabricante del adhesivo y pautas de la norma ASTM D 1084-08 (2008).

\section{Proceso de encolado y preparación de probetas}

\section{Encolado de listones}

El adhesivo PVA se aplicó de manera uniforme sobre una superficie total conformada por 5 listones de dimensiones de $5 \times 20 \mathrm{~cm}$. Se utilizaron tres extendidos del adhesivo (150, 200 y $250 \mathrm{~g} / \mathrm{m}^{2}$ ) con seis (6) minutos de exposición (abierto) y doce (12) minutos de ensamble (cerrado).

\begin{tabular}{|c|l|c|c|}
\hline & \multicolumn{1}{|c|}{ Variable } & Unidades & Promedio \\
\hline \multirow{5}{*}{ Ambiental } & Temperatura ambiental & ${ }^{\circ} \mathrm{C}$ & 15,70 \\
\cline { 2 - 4 } & Humedad relativa & $\%$ & 84 \\
\hline \multirow{5}{*}{ Encolado } & Tiempo de exposición & minutos & 6 \\
\cline { 2 - 4 } & Tiempo de ensamble & minutos & 12 \\
\cline { 2 - 4 } & Tiempo bajo presión & horas & 6 \\
\cline { 2 - 4 } & Extendido de cola & $\mathrm{g} / \mathrm{m}^{2}$ & $\begin{array}{c}150,200 \\
\mathrm{y} 250\end{array}$ \\
\cline { 2 - 4 } & Presión de prensado & $\mathrm{kg} / \mathrm{cm}^{2}$ & $5 \mathrm{y} 10$ \\
\cline { 2 - 4 } & Tipo de aplicación & $\mathrm{doble}$ & 2 caras \\
\cline { 2 - 4 } & $\begin{array}{l}\text { Contenido de humedad } \\
\text { de equilibrio de la } \\
\text { madera }\end{array}$ & $\%$ & 15,27 \\
\hline
\end{tabular}

Cuadro 2. Condiciones del proceso de encolado.



Figura 1. Distribución de los puntos de lectura de la rugosidad superficial.

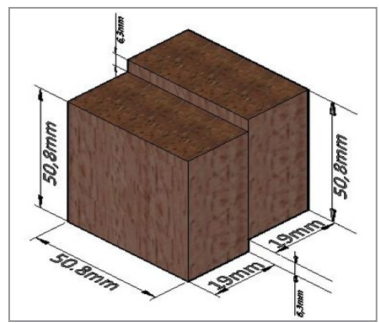

Figura 2. Dimensiones de las probetas para el ensayo de cizallamiento en la línea de cola.

\section{Prensado de listones}

Se prensaron los listones en la Prensa CIMPA con presiones de prensado de 5 y $10 \mathrm{~kg} /$ $\mathrm{cm}^{2}$ por un tiempo de 6 horas. Cada presión de prensado se incrementó en $0,5 \mathrm{~kg} / \mathrm{cm}^{2}$, debido a la pérdida de presión por el reacomodo de los listones.

\section{Acondicionado de listones}

Los listones prensados se acondicionaron a las condiciones ambientales del laboratorio de ensayo, por un periodo de 7 días antes de la preparación de las probetas. En el Cuadro 2 se muestran las condiciones ambientales durante el ensayo y las características del proceso de encolado.

\section{Preparación de las probetas}

Se procedió al corte y dimensionamiento final de las probetas, obteniéndose probetas con las medidas especificadas en la norma ASTM-D-905-03 (2003) (Figura 2).

\section{Ensayo de resistencia al cizallamiento en la línea de cola}

Los ensayos se realizaron en el Laboratorio de Propiedades Físicas y Mecánicas de la Madera, de acuerdo a la norma ASTM-D-905-03 (2003), empleando la Prensa Universal "Tinius Olsen" con una velocidad de carga ajustada a 
$0,4 \mathrm{~mm} / \mathrm{min}$. Se determinó la carga máxima al esfuerzo de cizallamiento en la línea de cola.

Determinación de resistencia al cizallamiento en la línea de cola

Se determinó la resistencia al cizallamiento a través de la siguiente relación:

$$
C z=P / A
$$

Dónde: $\mathrm{Cz}$ es la resistencia al cizallamiento en $\mathrm{kg} / \mathrm{cm}^{2}$; P es la carga máxima registrada en $\mathrm{kg}$ y A es el área encolada en la probeta $\mathrm{cm}^{2}$.

\section{Estimación del porcentaje de madera fallada}

Se realizó al escaneo de la superficie fallada en cada probeta ensayada, con la finalidad de obtener imágenes que permitan delimitar el área de madera fallada. Para la estimación de la superficie fallada por probeta se empleó el programa AutoCad 2013, y la determinación del porcentaje de madera fallada se realizó con la siguiente relación:

\section{Porcentaje de madera fallada $=$ Superficie de madera fallada ${ }^{\star} 100 /$ Superficie encolada}

\section{Evaluación de la unión encolada}

Se realizó la evaluación de la unión encolada (Cuadro 3), combinando la resistencia relativa (relación de la resistencia de la unión encolada entre la resistencia de la madera sólida, expresada en porcentaje) con el porcentaje de madera fallada.

\section{Diseño experimental}

Se aplicó un Diseño Completamente al Azar (DCA) con un arreglo factorial de $2 \mathrm{~A} \times 3 \mathrm{~B}$ con 10 repeticiones por tratamiento, de acuerdo al siguiente esquema:

Unidad experimental: probetas de madera tornillo procedentes de tratamientos silviculturales de campo abierto y fajas de $5 \mathrm{~m}$.

Variable observada: carga máxima al cizallamiento en la línea de cola.

Nivel de significación: 95 \% de confianza con un $\alpha=0.05$

\section{Factores:}

- Factor A: presión de prensado $\left(5\right.$ y $\left.10 \mathrm{~kg} / \mathrm{cm}^{2}\right)$

- Factor B: extendido de cola $\left(150,200,250 \mathrm{~g} / \mathrm{m}^{2}\right)$

\section{Resultados}

\section{Evaluaciones preliminares}

En el Cuadro 4 se indican los resultados de las evaluaciones preliminares para la madera $\mathrm{y}$ adhesivo empleados en la presente investigación.

En relación a la madera, el contenido de humedad promedio es inferior al contenido de equilibrio higroscópico registrado para el periodo de la fase experimental.

\begin{tabular}{|c|c|c|c|c|c|}
\hline \multirow{2}{*}{$\begin{array}{c}\text { Madera } \\
\text { fallada (\%) }\end{array}$} & \multicolumn{5}{|c|}{ Resistencia relativa (\%) } \\
\cline { 2 - 6 } & $\begin{array}{c}\text { Excelente } \\
\mathbf{( 9 1 - 1 0 0 )}\end{array}$ & $\begin{array}{c}\text { Muy Buena } \\
\mathbf{( 8 1 - 9 0 )}\end{array}$ & $\begin{array}{c}\text { Buena } \\
\mathbf{( 7 1 - 8 0 )}\end{array}$ & $\begin{array}{c}\text { Regular } \\
\mathbf{( 6 1 - 7 0 )}\end{array}$ & $\begin{array}{c}\text { Mala } \\
(<\mathbf{6 0 )}\end{array}$ \\
\hline $\begin{array}{c}\text { Excelente } \\
(\mathbf{8 1 - 1 0 0 )}\end{array}$ & Excelente & Excelente & $\begin{array}{c}\text { Muy } \\
\text { Buena }\end{array}$ & $\begin{array}{c}\text { Muy } \\
\text { Buena }\end{array}$ & Buena \\
\hline $\begin{array}{c}\text { Muy Buena } \\
\mathbf{( 6 1 - 8 0 )}\end{array}$ & Excelente & $\begin{array}{c}\text { Muy } \\
\text { Buena }\end{array}$ & $\begin{array}{c}\text { Muy } \\
\text { Buena }\end{array}$ & Buena & Buena \\
\hline $\begin{array}{c}\text { Buena } \\
\mathbf{( 4 1 - 6 0 )}\end{array}$ & Excelente & $\begin{array}{c}\text { Muy } \\
\text { Buena }\end{array}$ & Buena & Regular & $\begin{array}{c}\text { Regu- } \\
\text { lar }\end{array}$ \\
\hline $\begin{array}{c}\text { Regular } \\
(\mathbf{2 1 - 4 0 )}\end{array}$ & $\begin{array}{c}\text { Muy } \\
\text { Buena }\end{array}$ & $\begin{array}{c}\text { Muy } \\
\text { Buena }\end{array}$ & Regular & Regular & Mala \\
\hline Mala (<20) & $\begin{array}{c}\text { Muy } \\
\text { Buena }\end{array}$ & Buena & Regular & Mala & Mala \\
\hline
\end{tabular}

Cuadro 3. Tabla para evaluación de uniones encoladas: resistencia relativa vs. madera fallada.

\begin{tabular}{|c|c|c|c|}
\hline \multicolumn{2}{|c|}{ Descripción } & Unidad & Promedio \\
\hline \multirow{3}{*}{ Madera } & Contenido de humedad & $\%$ & 15,00 \\
\cline { 2 - 4 } & $\begin{array}{c}\text { Porcentaje de } \\
\text { extractivos }\end{array}$ & $\%$ & 5,20 \\
\hline \multirow{2}{*}{ Adhesivos } & Viscosidad & $\mathrm{Cps}$ & 14500,00 \\
\cline { 2 - 4 } & Contenido de sólidos & $\%$ & 42,30 \\
\hline
\end{tabular}

Cuadro 4. Evaluaciones preliminares en la madera y el adhesivo PVA.

\begin{tabular}{|c|c|}
\hline Fuente de variabilidad & Significancia $(\boldsymbol{\alpha}=\mathbf{0 . 0 5})$ \\
\hline Presión & No significativo \\
\hline Extendido & No significativo \\
\hline Interacción presión-extendido & No significativo \\
\hline
\end{tabular}

tencia al cizallamiento en la línea de cola para madera tornillo en fajas a $5 \mathrm{~m}$. 
Con respecto al adhesivo PVA, los resultados están dentro del rango con las especificaciones técnicas indicadas por el fabricante. El contenido de sólidos de 42,30 por ciento, es inferior al indicado en la norma NTP 260.015 (2012), que indica que el porcentaje mínimo de sólidos para elaboración de mobiliario escolar es de 48 por ciento

\section{Resistencia al cizallamiento en la línea de cola}

En las Figuras 3 y 4 se muestran los valores promedios de resistencia al cizallamiento y de porcentaje de madera fallada para la madera tornillo según cada tratamiento silvicultural.

Para la madera tornillo en fajas (Figura 3), el tratamiento con $10 \mathrm{~kg} / \mathrm{cm}^{2}$ de presión y $150 \mathrm{~g} /$ $\mathrm{m}^{2}$ de extendido, registra los mayores valores de resistencia al cizallamiento y porcentaje de madera fallada, con $80,33 \mathrm{~kg} / \mathrm{cm}^{2}$ y 57,86 por ciento, respectivamente.
La madera tornillo en campo abierto (Figura 4) con el tratamiento de $10 \mathrm{~kg} / \mathrm{cm}^{2}$ de presión y $250 \mathrm{~g} / \mathrm{m}^{2}$ de extendido, registra los mayores valores de resistencia al cizallamiento y porcentaje de madera fallada, con $83,70 \mathrm{~kg} / \mathrm{cm}^{2}$ y 33,37 por ciento, respectivamente.

\section{Diseño experimental}

Fajas de 5 metros

En el Cuadro 5 se observa que no existen diferencias significativas entre las presiones de encolado, por lo cual, se puede trabajar indistintamente en cualquier nivel de presión obteniendo similares resistencias al cizallamiento en la línea de cola. De la misma manera, se encontró suficiente evidencia estadística para afirmar que los diferentes extendidos del adhesivo proporcionan parecidas resistencias al cizallamiento.


Figura 3. Promedios de resistencia al cizallamiento de la línea de cola y de madera fallada para la madera tornitlo en fajas de $5 \mathrm{~m}$ según tratamiento.

Figura 4. Promedios de resistencia al cizallamiento de la línea de cola y de madera fallada para la madera de tornillo en campo abierto según tratamiento. 
En cuanto a la interacción, no existe efecto combinado de la presión de encolado y extendido sobre la resistencia al cizallamiento en la línea de cola. De esta manera, el tratamiento con $10 \mathrm{~kg} / \mathrm{cm}^{2}$ y $150 \mathrm{~g} / \mathrm{m}^{2}$ representa la mejor condición de encolado, debido al mayor valor de resistencia registrada (Figura 3) y la menor cantidad de adhesivo empleado.

\section{Campo abierto}

En el Cuadro 6 se muestra el análisis de varianza de la resistencia al cizallamiento en la línea de cola para madera tornillo en campo abierto. Este análisis demuestra que es significativa la varianza en la presión de encolado y extendido, por lo tanto, hay suficiente evidencia estadística para afirmar que alguna de las presiones y extendidos ensayados, proporciona diferente resistencia al cizallamiento en la línea de cola.

En el Cuadro 7 se muestran las comparaciones entre tratamientos para el ensayo de resistencia al cizallamiento en la línea de cola según la Prueba de Tukey. El tratamiento con $10 \mathrm{~kg} / \mathrm{cm}^{2}$ de presión de encolado y $250 \mathrm{~g} / \mathrm{m}^{2}$ de extendido proporciona diferente resistencia al cizallamiento en la línea de cola en comparación con los tratamientos con $5 \mathrm{~kg} / \mathrm{cm}^{2}-150 \mathrm{~g} /$ $\mathrm{m}^{2}$ y $5 \mathrm{~kg} / \mathrm{cm}^{2}-200 \mathrm{~g} / \mathrm{m}^{2}$. De la misma manera, los tratamientos con $5 \mathrm{~kg} / \mathrm{cm}^{2}-200 \mathrm{~g} / \mathrm{m}^{2}$ y 10 $\mathrm{kg} / \mathrm{cm}^{2}-200 \mathrm{~g} / \mathrm{m}^{2}$, representan desiguales resistencias al cizallamiento en la línea de cola. En las otras comparaciones, los tratamientos generan resistencias al cizallamiento en la línea de cola con valores similares.

De esta manera, el tratamiento con $10 \mathrm{~kg} /$ $\mathrm{cm}^{2}-150 \mathrm{~g} / \mathrm{m}^{2}$ representa la mejor condición de encolado para madera tornillo en campo abierto, debido a que la evidencia estadística indica que genera similar resistencia a la condición $10 \mathrm{~kg} / \mathrm{cm}^{2}-250 \mathrm{~g} / \mathrm{m}^{2}$ (Cuadro 7). Cabe resaltar que el tratamiento con $10 \mathrm{~kg} / \mathrm{cm}^{2}-250 \mathrm{~g} /$ $\mathrm{m}^{2}$, es el que registra el mayor valor de resistencia al cizallamiento en la línea de cola (Figura 4). Adicionalmente, el tratamiento con $10 \mathrm{~kg} /$ $\mathrm{cm}^{2}-150 \mathrm{~g} / \mathrm{m}^{2}$ emplea una menor cantidad de adhesivo.

\section{Evaluación de la unión encolada}

Según el Cuadro 8 para la madera tornillo en fajas de $5 \mathrm{~m}$, las uniones encoladas varían de buenas a muy buenas. En el caso de campo abierto, la variabilidad es mayor obteniéndose uniones encoladas que califican de malas a muy buenas.

Cabe resaltar que el tratamiento con $10 \mathrm{~kg} /$ $\mathrm{cm}^{2}$ de presión y $150 \mathrm{~g} / \mathrm{m}^{2}$ de extendido, para fajas de $5 \mathrm{~m}$, y $10 \mathrm{~kg} / \mathrm{cm}^{2}$ de presión y $200 \mathrm{~g} /$ $\mathrm{m}^{2}$ de extendido para campo abierto, reportan uniones encoladas muy buenas; garantizando

\begin{tabular}{|c|c|}
\hline Fuente de variabilidad & Significancia $(\boldsymbol{\alpha}=\mathbf{0 . 0 5})$ \\
\hline Presión & Significativo \\
\hline Extendido & Significativo \\
\hline Interacción presión-extendido & No significativo \\
\hline
\end{tabular}

Cuadro 6. Análisis de variancia para el ensayo de resistencia al cizallamiento en la línea de cola para madera tornillo en campo abierto.

\begin{tabular}{|c|c|}
\hline $\begin{array}{c}\text { Comparación (Presión en } \mathbf{k g} / \\
\left.\mathbf{c m}^{2} \mathbf{y} \text { Extendido en } \mathbf{g} / \mathbf{c m}^{2}\right)\end{array}$ & $\begin{array}{c}\text { Significancia } \\
(\boldsymbol{\alpha}=\mathbf{0 . 0 5})\end{array}$ \\
\hline 5 y $150-10$ y 250 & Significativo \\
\hline 5 y $200-10$ y 200 & Significativo \\
\hline 5 y $200-10$ y 250 & Significativo \\
\hline
\end{tabular}

Cuadro 7. Prueba de Tukey para el ensayo de resistencia al cizallamiento en la línea de cola para madera tornillo en campo abierto.

\begin{tabular}{|c|c|c|}
\hline \multirow{2}{*}{$\begin{array}{c}\text { Tratamiento } \\
\text { (Presión en kg/cm² y } \\
\text { Extendido en } \mathbf{g} / \mathrm{m}^{2} \text { ) }\end{array}$} & \multicolumn{2}{|c|}{ Calificación } \\
\hline & Fajas a 5m & Campo abierto \\
\hline 5 y 150 & Buena & Regular \\
\hline 5 y 200 & Buena & Mala \\
\hline 5 y 250 & Muy Buena & Buena \\
\hline 10 y 150 & Muy Buena & Buena \\
\hline 10 y 200 & Muy Buena & Muy Buena \\
\hline 10 y 250 & Muy Buena & Muy Buena \\
\hline
\end{tabular}

Cuadro 8. Evaluación de las uniones encoladas para madera tornillo según tratamiento silvicultural.

\begin{tabular}{|c|c|c|}
\hline Tratamiento silvicultural & Promedio $(\boldsymbol{\mu m})$ & $\mathbf{C V}(\%)$ \\
\hline Fajas a 5 m (22 años) & 13,90 & 28,20 \\
\hline Campo abierto (20 años) & 13,20 & 25 \\
\hline
\end{tabular}

Cuadro 9. Valores de rugosidad superficial para madera tornillo según sistema silvicultural. 
menores consumos de adhesivo y mayores valores de resistencia al cizallamiento y porcentajes de madera fallada.

\section{Rugosidad superficial}

Según el Cuadro 9, para ambos tratamientos silviculturales, se registra una rugosidad superficial superior a la reportada por Gonzales (2006) (con 11,35 $\mu \mathrm{m}$ ), para madera cepillada de pino chuncho (Schizolobium amazonicum). Como las maderas de pino chuncho y tornillo poseen algunas características anatómicas similares (grano entrecruzado y textura gruesa), la diferencia puede explicarse con el hecho que, en la presente investigación, las superficies a encolar no fueron cepilladas. $\mathrm{Al}$ respecto, Meléndez y Bustamante (2003) afirman que la rugosidad superficial depende en gran medida de varios factores, entre los cuales destacan: la estructura anatómica, el contenido de humedad, las particularidades de la madera de ciertas especies y árboles, el número de grano de lija, la forma geométrica de las piezas, la velocidad de corte y el afilado de los elementos de corte.

\section{Discusión}

El porcentaje de extractivos determinado con etanol es superior al registrado para madera tornillo procedente de bosques naturales (Bueno 1978). Al respecto, Leal et al. (2011) afirma que la cantidad de extractivos se incrementa con la edad del árbol debido al proceso de duraminización, en donde la madera cosechada en turnos cortos tendrá menor durabilidad que aquella de turnos largos. El menor valor registrado por Bueno (1978) puede estar relacionado con diferencias en la localización geográfica, condiciones de crecimiento, de clima y de sitio (Salazar y Pérez 1998).

Cabe resaltar que el promedio de resistencia al cizallamiento en el presente estudio representa el 88,4 por ciento de la resistencia al cizallamiento en la madera solida reportada por Villa (2009). El promedio de resistencia al cizallamiento para este tratamiento representa el 97,50 por ciento de la resistencia al cizallamiento en la madera solida reportada por Vega (2011).
Al comparar la resistencia promedio con estudios similares en maderas de Bombacáceas y la yanavara (Pollalesta discolor), se observa que los promedios de resistencia en fajas de $5 \mathrm{~m}$ y campo abierto se encuentran entre los valores registrados por Brandán (1991) $\left(45 \mathrm{~kg} / \mathrm{cm}^{2}\right)$ y Gonzales (2006) $\left(133,13 \mathrm{~kg} / \mathrm{cm}^{2}\right)$; esto permite inferir que la resistencia en la unión encolada depende de las propiedades físico-mecánicas de las maderas a unir, tal como también lo afirma López (1976).

De la misma manera, para la madera proveniente de ambos tratamientos silviculturales, se percibe una tendencia al incremento de la resistencia al cizallamiento a medida que aumentan la presión de encolado y el extendido del adhesivo. Al respecto, Frihart y Hunt (2010) afirman que una presión cercana a $7 \mathrm{~kg} / \mathrm{cm}^{2}$ es adecuada para maderas de baja densidad y Carrasco citado por Viveros (2007), recomienda una cantidad de adhesivo óptima entre 150 a $200 \mathrm{~g} / \mathrm{m}^{2}$.

Según Villa (2009), la madera de tornillo en fajas de 22 años es de baja densidad y dureza, esto explica que se obtengan aceptables uniones encoladas aún con menores presiones de prensado. Al respecto, Frihart y Hunt (2010) afirman que las bajas presiones de prensado son adecuadas para maderas de baja densidad, porque las superficies se acomodan fácilmente una con otra, asegurando el íntimo contacto entre el adhesivo y la madera.

Según Vega (2011), la madera de tornillo en campo abierto con 20 años presenta una densidad y dureza superior a la de fajas con 22 años, esto explica que sea necesario aplicar mayores presiones de prensado para obtener uniones encoladas aceptables. Al respecto, Frihart y Hunt (2010) afirman que mayores presiones de prensado son requeridas para maderas de mayor densidad, las cuales son más difíciles de comprimir. De la misma manera, Takahashi (1967) indica que una mayor dureza de lados dificulta la aplicación uniforme de la presión de prensado, recomendando el encolado de superficies lisas y libres de ondulaciones o irregularidades. 
Con relación a la presión de encolado, Frihart y Hunt (2010) señalan que una presión cercana a $7 \mathrm{~kg} / \mathrm{cm}^{2}$ es adecuada para maderas de baja densidad. Respecto al extendido de la cola, Carrasco citado por Viveros (2007) recomienda una cantidad óptima entre 150 a $200 \mathrm{~g} / \mathrm{m}^{2}$. El mismo autor afirma que las líneas de cola de poco espesor, además de ahorrar adhesivo, otorgan una mayor resistencia en la unión, menos riesgos de coloraciones y un fraguado más rápido.

Es posible que la menor densidad básica de la madera tornillo en fajas a $5 \mathrm{~m}$, represente un mayor volumen de espacio libre que permita la entrada del adhesivo, esto podría permitir una mejor adhesión mecánica, tal como también se afirma en el Foro Forestal Maderero de Bosque y Madera (2004).

De la misma manera, los mayores valores de dureza de lados y compresión perpendicular en la madera tornillo de campo abierto pueden contribuir a una mayor dificultad en el prensado de las uniones, debido a que se requieren de mayores presiones para dominar las irregularidades de la superficie encolada. Al respecto, Takahashi (1967) afirma que la dureza de lados y altos valores de compresión perpendicular generan dificultad para la aplicación uniforme de la presión e indican la necesidad de encolar superficies lisas y libres de ondulaciones o irregularidades.

\section{Conclusiones}

La resistencia al cizallamiento en la línea de cola utilizando adhesivo PVA en madera tornillo de fajas y campo abierto alcanza valores ligeramente inferiores a los de la madera sólida.

El incremento de la presión de prensado y el extendido de cola tienen un efecto favorable en la resistencia al cizallamiento en madera tornillo de campo abierto.

El incremento de la presión de prensado y el extendido de cola no tienen influencia sobre la resistencia al cizallamiento de madera tornillo en fajas de $5 \mathrm{~m}$.

Las uniones encoladas de mejor calidad en madera tornillo son obtenidas con una presión de prensado de $10 \mathrm{~kg} / \mathrm{cm}^{2}$, y con un extendido de $150 \mathrm{~g} / \mathrm{m}^{2}$ para fajas de $5 \mathrm{~m}$ y $200 \mathrm{~g} / \mathrm{m}^{2}$ para campo abierto.

\section{Bibliografía}

Acevedo, M. 1973. Propiedades físico-mecánicas y resistencia al cizallamiento de la línea de cola de la Tabebuia capitata (Bur y Sch) Sandw. Tesis para Optar el Título de Ingeniero Forestal. Lima, Perú. Universidad Nacional Agraria La Molina. 107 p.

Aróstegui, A. 1982. Recopilación de estudios tecnológicos de maderas peruanas. Lima, Perú, PNUD/FAO/PER/71/511.57 p. (Documento de trabajo No 2).

ASTM (American Society for Testing and Materials, US). 2003. ASTM Designation: D 905 - 03 "Standard Method of Test for Strength Properties of Adhesive Bonds in Shear by Compression Loading". 5 p.

ASTM (American Society for Testing and Materials, US) D 907-08. 2008. Standard Terminology of Adhesives. USA. $12 \mathrm{p}$

ASTM (American Society for Testing and Materials, US) D 5536-94. 2004. Standard Practice for Sampling Forest Trees for Determination of Clear Word Properties. USA. 9 p.

ASTM (American Society for Testing and Materials, US) D 1489-97. 2004. Standard Test Method for Nonvolatile Content of Aqueous Adhesives. USA. 2 p.

ASTM (American Society for Testing and Materials, US) D 1084-08. 2008. Standard Test Method for Viscosity of Adhesivos. USA. 5 p.

Brandán, S. 1991. Uniones encoladas con PVA, tres métodos de prensado y tres extendidos en cuatro maderas de Bombacaceae. Tesis para Optar el Título de Ingeniero Forestal. Lima, Perú. Universidad Nacional Agraria La Molina. $84 \mathrm{p}$.

Bueno, J. 1978. Estudio de posibilidades industriales de maderas nacionales para fabricación de pulpa para papel. Lima, Perú. 223 p.

Flores, Y; Ugalde, L; Galloway, G; Carrera, F. 2004. Crecimiento y productividad de plant- 
aciones de seis especies forestales nativas de 20 años de edad en el bosque Alexander Von Humbolt, Amazonia Peruana. Recursos Naturales y Ambiente. Lima, Perú. 111-121 p.

Foro Forestal Maderero de bosque y madera. 2004. Adhesivos (en línea). Consultado junio.2014. Disponible en: http://www.ffm.cl/ viewtopic.php? $\mathrm{t}=4$

Frihart, ChR; Hunt, ChG. 2010. Adhesives with Wood Materials Bond Formation and Performance. Capítulo 10. En: Wood Handbook. Wood as an Engineering Material. Forest Products Laboratory. United States Department of Agriculture Forest Service. Madison, Wisconsin. 24 p.

Gonzales, M. 2006. Efecto de dos métodos de cepillado en la resistencia al cizallamiento de la línea de cola de dos especies de bosques secundarios. Tesis para Optar el Título de Ingeniero Forestal. Lima, Perú. Universidad Nacional Agraria La Molina. 71 p.

Leal, LE; Juárez, V; Terán, M. 2011. Composición química de la madera Eucalyptus grandis Hill ex Maiden procedente de Finca Las Maravillas, Departamento de Orán, Salta. Revista de Ciencias Forestales - Quebracho 19 (1-2).

López, MA. 1976. Resistencia y comportamiento al cizallamiento de la línea de cola de resorcinol-formaldehido en doce especies forestales. Tesis para Optar el Título de Ingeniero Forestal. Lima, Perú. UNALM. 77 p.

Meléndez, CA; Bustamante, GN. 2003. Evaluación de rugosidad superficial en madera cepillada y lijada de 6 especies forestales. UNALM. Departamento de industrias forestales. Lima, Perú.

NTP (Norma Técnica Peruana) 251.010. 2004. Método para determinar el contenido de humedad. 2a. ed. 13 p.

NTP (Norma Técnica Peruana) 260.015. 2012. Mobiliario escolar para instituciones educativas. Requisitos. 3a. ed.

Salazar, AH; Pérez, JH. 1998. Determinación de componentes químicos de la madera cinco especies de encino del estado de Puebla. Revista Madera y Bosque 4 (2): 79-93.
Takahashi, L. 1967. Resistencia al cizallamiento de la línea de cola, en quinilla colorada (humiriastrum excelsum Ducke). Revista Forestal del Perú 1 (2): 22-28.

TAPPI (Technical Association of the Pulp and Paper Industry). 1997. T 204 cm-97 "Solvent extractives of wood and pulp". $4 \mathrm{p}$.

Vega, N. 2011. Evaluación de las propiedades físico mecánicas de Cedrelinga cateniformis (Ducke) Ducke procedente de plantaciones de 20 años del Bosque Nacional Alexander von Humboldt Ucayali (Perú). Tesis para Optar el Título de Ingeniero Forestal. Lima, Perú. Universidad Nacional Agraria La Molina. 117 p.

Villa, B. 2009. Propiedades físico mecánicas de la especie Cedrelinga cateniformis Ducke provenientes de plantaciones instaladas en la Estación Experimental Alexander von Humboldt. Tesis para Optar el Título de Ingeniero Forestal. Lima, Perú. Universidad Nacional Agraria La Molina. 133 p.

Viveros, M. 2007. Efecto del desgaste del cuchillo en la calidad de la unión adhesiva. Tesis para Optar el Título de Ingeniero civil en industrias forestales. Concepción, Chile. Universidad del Bio-Bio. 57 p. Consultado junio 2014. Disponible en: http://cybertesis.ubiobio. cl/tesis/2007/viveros_e/html/index-frames. html

Presentado: $14 / 02 / 2016$ Aceptado: 19/04/2016 